\title{
Informationsverluste bei sektorübergreifender Behandlung: Ursachen und Lösungen
}

Christoph Straub, Daniela Teichert, Karl Blum, Daniel Grandt

\section{Informationsverluste bei der Krankenhaus-Einweisung oder -Entlassung können die Patienten erheblich gefährden. Inwieweit Fortschritte bei der Digitalisierung dem entgegenwirken können, ist seit längerem Gegenstand der Diskussion. Dieser Bei- trag beschreibt ein öffentlich gefördertes Projekt zur transsektoralen Optimierung der Patientensicherheit und stellt erste Ergebnisse einer aktuellen Umfrage bei}

\section{1 deutschen Kliniken vor.}

„Die Digitalisierung kann die Gesundheitsversorgung durch ein sektorübergreifendes und zeitnahes Vorliegen von Behandlungsinformationen verbessern und die Patientensicherheit erhöhen, “ so der Sachverständigenrat für die Entwicklung im Gesundheitswesen (SVR Gesundheit) [1]. Er sieht Deutschland weit hinter anderen Ländern bei der Digitalisierung, der Verarbeitung von Informationen und der sektorübergreifenden Kommunikation. In einem Sondergutachten stellte der SVR zuvor fest, dass „..bei der Einweisung von Patienten in den akut-stationären Bereich in 15\% der Fälle Beeinträchtigungen bei der klinischen Behandlung aufgrund unzureichender Informationen auftraten. In $4 \%$ der Fälle wurde eine unmittelbare Gefährdung von Patienten aufgrund von (intersektoralen) Informationsmängeln deutlich“ [2]. Ein durch Beschluss des Gemeinsamen Bundesausschusses mit 9,3 Millionen Euro aus dem Innovationsfonds gefördertes Projekt zur trans-sektoralen Optimierung der Patientensicherheit („TOP“) hat das Potenzial, diese schon lange beklagte, vermeidbare Patientengefährung zu verhindern und kann darüber hinaus sogar helfen, die Behandlungseffizienz in Krankenhäusern zu verbessern [3].

Mit Einverständnis des Patienten werden dem Arzt bei Krankenhausaufnahme Informationen zu allen dem Patienten verordneten und abgegebenen Arzneimitteln, zu bestehenden Erkrankungen, erfolgter Diagnostik, bisherigen Behandlungen, den ambulant behandelnden Ärzten und bisherigen Krankenhausbehandlungen zur Verfügung gestellt. Dabei handelt es sich um Informationen, die aus den Abrechnungsdaten der beteiligten Krankenkassen, der BARMER und der AOK Nordost, extrahiert und aggregiert werden und die dem Krankenhaus zudem ohne Zeitverzug über die Telematikinfrastruktur (TI) elektronisch und stukturiert zur Verfügung stehen. 14 Krankenhäuser in 6 Bundesländern, darunter 3 Universitätskliniken, nehmen an dem Projekt teil, in dem Kassenärztliche Vereinigungen, die Deutsche Gesellschaft für Innere Medizin (DGIM), mehr als 20 weitere Fachgesellschaften, der Verband der deutschen Krankenhausapotheker (ADKA) und das Deutsche Krankenhausinstitut (DKI) mitarbeiten.

Das DKI hat 2021 im Rahmen des Projektes eine Umfrage unter Krankenhäusern in Deutschland durchgeführt, um den Stand der Digitalisierung und der AMTS sowie eine Einschätzung der neuen Versorgungsform TOP aus Sicht der Krankenhäuser abzufragen. Die Antworten von 221 an der Umfrage teilnehmenden Kliniken, die bezüglich Größe, Trägerstatus und Lage eine recht repräsentative Stichprobe darstellen, weisen auf Ursachen für die Probleme des sektorübergreifenden Informationsaustausches hin, welche durch das Krankenhauszukunftsgesetz (KHZG) allein nicht gelöst werden können.

98 \% der Krankenhäuser wünschen sich, behandlungsrelevante Informationen, wie z. B. zur ambulanten Arzneimitteltherapie, elektronisch zu erhalten und weiterverarbeiten zu können. 96 \% würden diese Informationen auch gerne zum Entlasszeitpunkt wieder elektronisch dem weiterbehandelnden Arzt zuleiten. Realität aber ist, dass es in Deutschland keinen Standard für die Kodierung von Informationen zu Arzneimitteln und ihrer Dosierung gibt, der auch für komplexe Dosierungsschemata, wie z. B. im Krankenhaus üblich, geeignet ist. Informationsaustausch ist daher der Austausch von Papier - und Informationsdefizite sind die Folge und die Regel. Besonders betroffen sind die vulnerabelsten Patienten, die als Notfall ins Krankenhaus kommen ( $\triangleright$ Abb. 1).

Bei 4 von 5 Notfallpatienten fehlen für die Behandlung relevante Informationen, bei Elektivpatienten fehlen diese bei jedem 2. bis 3. Patienten. Informationsdefizite gefährden Patienten, weil sie zu falschen Behandlungsentscheidungen führen können. Studien zeigen, dass $64 \%$ der Patienten keine korrekten Angaben zu ihren Arzneimitteln machen können [4], bei Patienten mit 9 und 


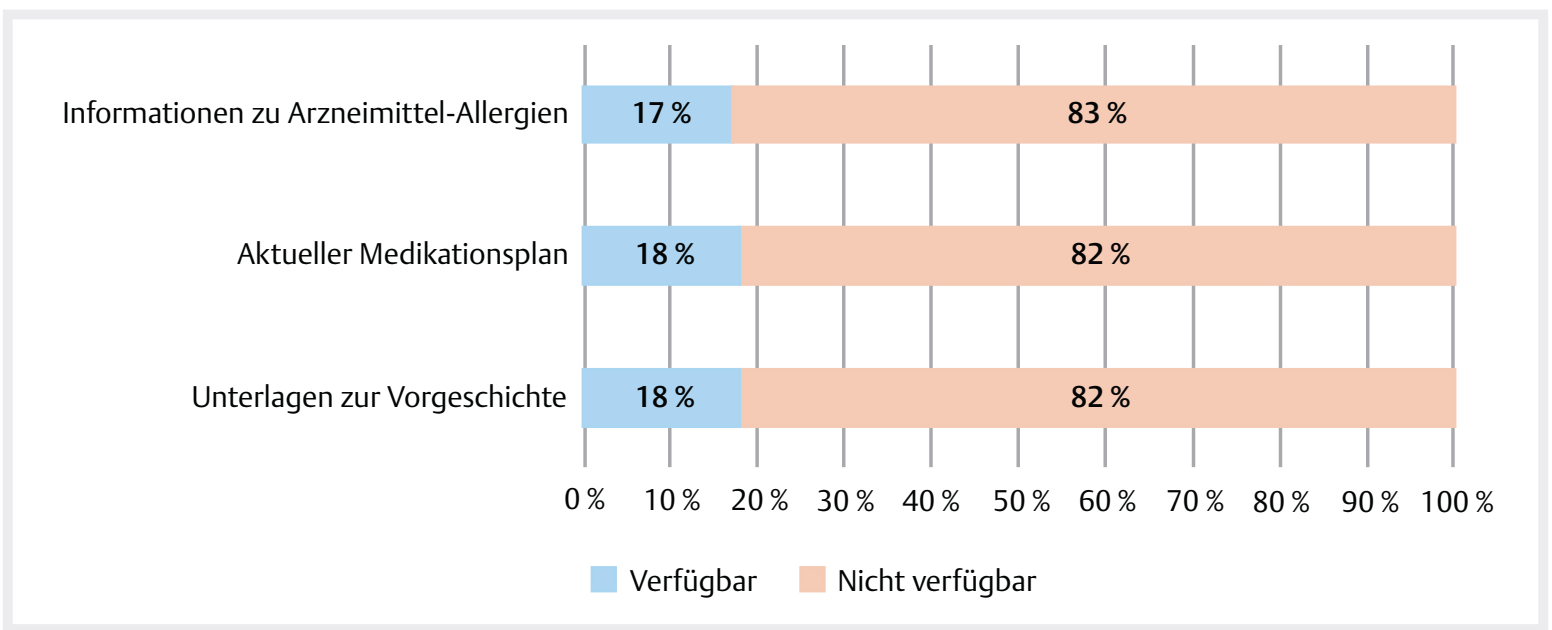

- Abb. 1 Verfügbarkeit für die Behandlung notwendiger Informationen für Notfall-Patienten bei Krankenhaus-Aufnahme. Quelle: Umfrage des DKI unter Krankenhäusern in Deutschland $(n=221)$.

- Tab. 1 Beurteilung des Nutzens der TOP-Intervention durch Krankenhäuser. Quelle: Umfrage des DKI unter Krankenhäusern in Deutschland $(n=221)$.

\begin{tabular}{|l|l|l|}
\hline $\begin{array}{l}\text { Wie beurteilen Sie den Nutzen der TOP-Maßnahmen für Ihre Patienten in Ihrem } \\
\text { Krankenhaus? }\end{array}$ & Sehr/ziemlich groß & Nicht sehr groß \\
\hline $\begin{array}{l}\text { Sichere und sofortige Verfügbarkeit von geprüften Informationen in Bezug auf ... } \\
\text { - die ambulante Arzneimitteltherapie }\end{array}$ & & \\
\hline - Vorerkrankungen (Diagnosen) & $100 \%$ & $0 \%$ \\
\hline - ambulant behandelnde Ärzte & $96 \%$ & $4 \%$ \\
\hline die Erleichterung der Erstellung des BMP & $93 \%$ & $7 \%$ \\
\hline elektronische Unterstützung bei der AMTS-Prüfung während der Aufnahme & $91 \%$ & $9 \%$ \\
\hline automatisiert erstellte Risikoanalyse zur AMTS bei Aufnahme & $96 \%$ & $4 \%$ \\
\hline den Einsatz von Apothekern bei Aufnahme & $93 \%$ & $7 \%$ \\
\hline
\end{tabular}

mehr Arzneimitteln können dies noch nicht einmal 10\% [5]. Das Problem ist relevant: In einer prospektiven Untersuchungen waren $43 \%$ der Patienten bei Krankenhausaufnahme von einem Fehler bei der Medikationsanamnese betroffen [6]. Bei $75 \%$ multimorbider Patienten kam es durch Anamnesefehler zu Fehlern bei der Weiterführung ambulanter Arzneimitteltherapie im Krankenhaus [7]. $75 \%$ der Medikationsfehler im Krankenhaus gehen auf Verordnungsfehler bei der stationären Aufnahme zurück [8]. Fehler im Bereich der Medikationsanamnese bei Aufnahme sind auch eine wichtige Ursache fehlerhafter Therapie-Empfehlungen bei der Entlassung [9].

Es ist daher nicht erstaunlich, dass laut der Befragung $100 \%$ der Krankenhäuser die Bereitstellung valider behandlungsrelevanter Informationen zum Patienten bei Aufnahme - wie in TOP realisiert - wünschen. Auch Hinweise auf potenziell vermeidbare Risiken der Arzneimitteltherapie zur Unterstützung des Arztes - ebenfalls in
TOP realisiert - wünschen sich $96 \%$ der Krankenhäuser $(\triangleright$ Tab. 1).

Nach Einschätzung der Krankenhäuser profitiert aber nicht nur der Patient von der neuen Versorgungsform TOP, sondern auch das Krankenhaus. Im Durchschnitt beträgt der Aufwand für die Medikationsanamnese 14 Minuten pro Patient. Der Zeitbedarf für das Nachfordern und Besorgen fehlender Informationen beträgt sogar 20 Minuten pro Patient -ein Aufwand, der nach Einschätzung von $98 \%$ der Krankenhäuser durch die Nutzung von Informationen aus Krankenkassendaten relevant reduziert werden kann. Gerade in Zeiten des kaum kompensierbaren Mangels an ärztlichen und pflegerischen Fachkräften ist die zeitliche Entlastung dieser Mitarbeiter ein wichtiges Argument für die neue Versorgungsform. In Dänemark ist dies längst Standard: Der Arzt erhält bei Krankenhausaufnahme eine Übersicht über die Arzneimittel des Patienten, die zentral gespei- 


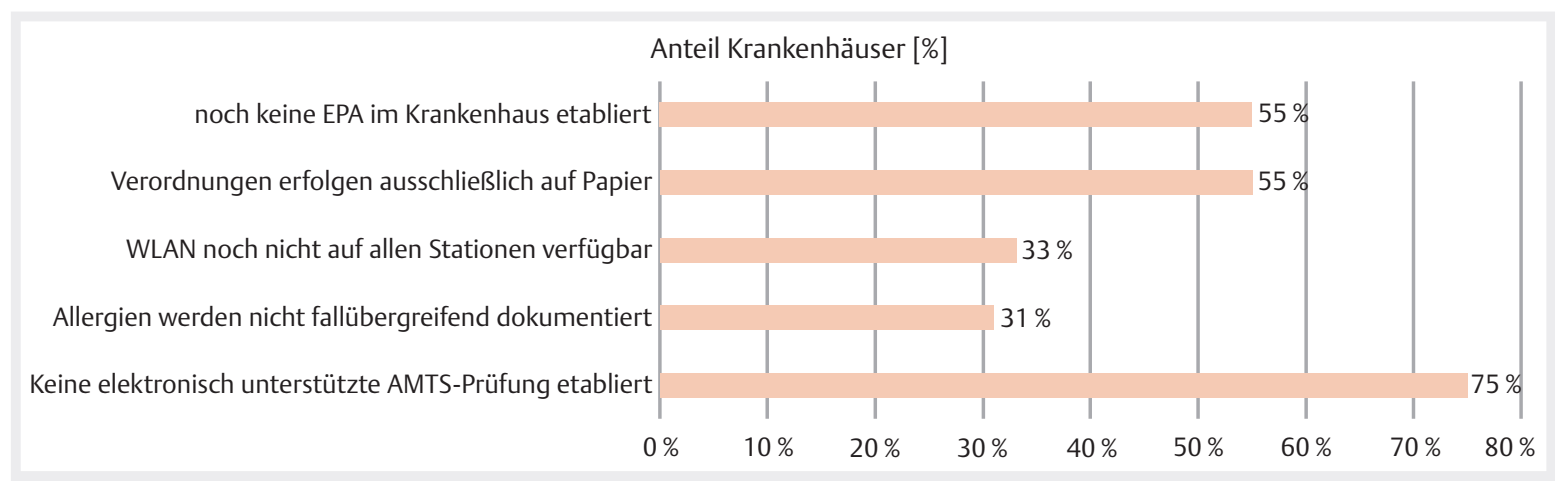

- Abb. 2 Stand der Digitalisierung der Krankenhäuser in Deutschland. Quelle: Umfrage des DKI unter Krankenhäusern in Deutschland $(n=221)$.

chert sind. Dass dies die Qualität der Medikationsanamnese relevant verbessert, ist durch Studien belegt [10], wie auch die Verringerung von Medikationsfehlern [11] und die Verringerung des Zeitaufwandes für die Informationsbeschaffung $[10,11]$. Eine Untersuchung zeigt, dass bei stationär aufgenommenen Patienten mit einem Fehler in der Aufnahmeanamnese die Nutzung von Krankenkassendaten Anamnesefehler und $61 \%$ der resultierenden Behandlungsfehler im Krankenhaus vermeiden kann [12].

TOP kann die Behandlungsqualität und Behandlungseffizienz flächendeckend verbessern, denn die erforderlichen Abrechnungsdaten sind für alle gesetzlich Krankenversicherten vorhanden und nutzbar. Neben der Verbesserung der Patientensicherheit werden Patienten vom Aufwand und der Verantwortung entlastet, den Informationsaustausch zwischen Niedergelassenen und Krankenhausärzten sicherzustellen. Mehr als $90 \%$ der befragten Patienten begrüßen das Angbebot und möchten es nutzen. „Warum gibt es das nicht schon lange? Das sollte selbstverständlich sein“, fasst ein Patient seine Einschätzung zusammen.

Hierzu sind aber Weichenstellungen auf Systemebene erforderlich. Ein einheitlicher Standard zur Abbildung der Arzneimitteltherapie, der auch im Krankenhaus funktioniert, ist eine unabdingbare Voraussetzung sektorübergreifender Interoperabilität von Informationen zur Arzneimitteltherapie und würde standardisierte und dann auch obligat vorzusehende Schnittstellen für Krankenhausinformationssysteme ermöglichen $(\triangleright$ Abb. 2).

Dass 87 \% der Krankenhäuser in der Umfrage einen einheitlichen Standard zur Abbildung der Arzneimitteltherapie für den ambulanten und stationären Sektor einfordern, ist verständlich. Notwendig ist ein Standard, der auch eine interoperable elektronische Dokumentation der Arzneimitteltherapie im Krankenhaus ermöglicht. In 55\% der Krankenhäuser erfolgt allerdings die Verordnung von Arzneimitteln heute noch ausschließlich handschriftlich.

\section{KERNAUSSAGEN}

- Die Nutzung von Routinedaten gesetzlicher Krankenkassen zur Behandlungsunterstützung verbessert die AMTS und die Behandlungseffizienz.

- Krankenhäuser wünschen den Sektor übergreifenden elektronischen Austausch von Informationen zum Patienten.

- Ein Standard zur Abbildung von Arzneimitteltherapie als Voraussetzung Sektor übergreifender Interoperabilität fehlt und wird dringlich benötigt.

\section{Interessenkonflikt}

Die Autorinnen/Autoren geben an, dass kein Interessenkonflikt besteht.

\section{Autorinnen/Autoren}

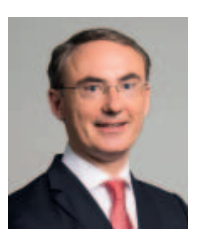

\section{Prof. Dr. Christoph Straub}

Vorstandsvorsitzender der BARMER Krankenkasse, Berlin

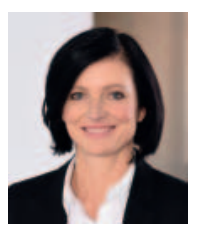

\section{Daniela Teichert}

Vorsitzende des Vorstandes der AOK Nordost Die Gesundheitskasse, Potsdam

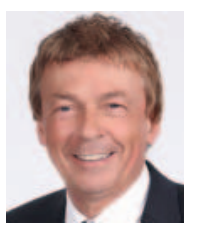

\section{Dr. Karl Blum}

Leiter Geschäftsbereich Forschung und Vorstand Deutsches Krankenhausinstitut e. V., Düsseldorf 


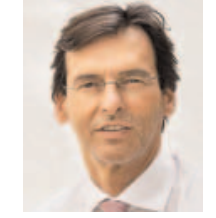

\section{Prof. Dr. Daniel Grandt}

Chefarzt der Klinik für Innere Medizin I, Klinikum Saarbrücken gGmbH, und Vorsitzender der Kommission Arzneimitteltherapiemanagement und Arzneimitteltherapiesicherheit der Deutschen Gesellschaft für Innere Medizin (DGIM)

\section{Korrespondenzadresse}

\section{Prof. Dr. Daniel Grandt}

Klinik für Innere Medizin I

Klinikum Saarbrücken gGmbh

Winterberg 1

66119 Saarbrücken

Deutschland

dgrandt@klinikum-saarbruecken.de

\section{Literatur}

[1] Gutachten des Sachverständigenrats im Gesundheitswesen 2021. Digitalisierung für Gesundheit: Ziele und Rahmenbedingungen eines dynamisch lernenden Gesundheitssystems. Im Internet (Letzter Zugriff am 18.11.2021): https://www.svrgesundheit.de/fileadmin/Gutachten/Gutachten2021 / SVR_ Gutachten_2021.pdf

[2] Sondergutachten des Sachverständigenrats im Gesundheitswesen 2021. Wettbewerb an der Schnittstelle zwischen ambulanter und stationärer Gesundheitsversorgung. Im Internet (Letzter Zugriff am 18.11.2021): https://dserver.bundestag.de/ btd/17/103/1710323.pdf

[3] Gemeinsamer Bundesausschuss. TOP - Transsektorale Optimierung der Patientensicherheit. Im Internet (Letzter Zugriff am 18.11.2021): https://innovationsfonds.g-ba.de/projekte/ neue-versorgungsformen/top-transsektorale-optimierung-derpatientensicherheit.367

[4] Meyer C, Stern M, Woolley W et al. How reliable are patientcompleted medication reconciliation forms compared with pharmacy lists? Am J Emerg Med 2012; 30 (7): 1048-1054

[5] Leal Hernández M, Abellán Alemán J, Casa Pina MT et al. Patients on multiple medication: do they know the right doses? Do they take their medications correctly? Aten Primaria 2004: 33 (8): 451-456
[6] de Andres-Lazaro AM, Sevilla-Sánchez D, Ortega-Romero Mdel $\mathrm{M}$ et al. [Accuracy in the medication history and reconciliation errors in the emergency department]. Med Clin (Barc) 2015; 145 (7): 288-293

[7] Alfaro-Lara ER, Santos-Ramos B, González-Méndez Al et al. Medication reconciliation on hospital admission in patients with multiple chronic diseases using a standardised methodology. Rev Esp Geriatr Gerontol 2013; 48 (3): 103-108

[8] Ashcroft DM, Lewis PJ, Tully MP et al. Prevalence, Nature, Severity and Risk Factors for Prescribing Errors in Hospital Inpatients: Prospective Study in 20 UK Hospitals. Drug Saf 2015; 38 (9): 833-843

[9] Delgado Sanchez O, Nicolás Picó ], Martínez López I et al. Reconciliation errors at admission and departure in old and polymedicated patients. Prospective, multicenter randomized study. Med Clin (Barc) 2009; 133 (19): 741-744

[10] Glintborg B, Poulsen HE, Dalhoff KP. The use of nationwide on-line prescription records improves the drug history in hospitalized patients. Br J. Clin. Pharmacol 2008; 65 (2): 265-269

[11] Phansalkar S, Her QL, Tucker AD et al. Impact of incorporating pharmacy claims data into electronic medication reconciliation. Am J Health Syst Pharm 2015; 72 (3): 212-217

[12] Pevnick JM, Palmer KL, Shane R et al. Potential benefit of electronic pharmacy claims data to prevent medication history errors and resultant inpatient order errors. J Am Med Inform Assoc 2016; 23 (5): 942-950

\section{Bibliografie}

Dtsch Med Wochenschr 2022; 147: 269-272

Online-Publikation: 14.2.2022

DOI $10.1055 / \mathrm{a}-1729-8798$

ISSN $0012-0472$

(c) 2022. The Author(s).

This is an open access article published by Thieme under the terms of the Creative Commons Attribution-NonDerivative-NonCommercial License, permitting copying and reproduction so long as the original work is given appropriate credit. Contents may not be used for commecial purposes, or adapted, remixed, transformed or built upon. (https://creativecommons.org/ licenses/by-nc-nd/4.0/)

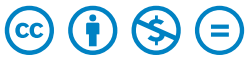

